Thorax (1962), 17, 310.

\title{
THE EFFECT OF SYMPATHECTOMY, VAGOTOMY, AND OESOPHAGEAL INTERRUPTION ON THE CANINE GASTRO-OESOPHAGEAL SPHINCTER
}

\author{
BY \\ RICHARD K. GREENWOOD,* JERRY F. SCHLEGEL, CHARLES F. CODE,
AND F. HENRY ELLIS, Jr.
}

From the Mayo Clinic and Mayo Foundation, Rochester, Minnesota, U.S.A.

The nature of the control of the gastrooesophageal sphincter is obscure. It has been thought to be under the regulation of the autonomic nervous system so that through the influences of the vagus and sympathetic nerves the resting tone is maintained and relaxation and contraction are initiated. Beyond this broad generalization, however, there is considerable divergence of opinion as to the precise function of the various nervous elements and their interrelationships.

The purpose of this investigation was to determine the effect of certain nerve and oesophageal interruption procedures on the resting tone of the sphincter and its relaxation and contraction during swallowing.

\section{REVIEW OF THE LiTERATURE}

A comprehensive review of the work of previous investigators in this field is given by Ingelfinger (1958) in his treatise on oesophageal motility. Most studies have involved stimulation or destruction of the vagus and sympathetic nerves. The usual methods of observation have been manometric estimations and radiological examination.

All workers agree that the vagus has some influence in the lower end of the oesophagus. Many believe that the vagus exerts both inhibitory and motor actions on the sphincter, and it has been suggested that these impulses travel by different routes. Openchowski (1889) postulated the existence of a distinct 'dilator cardiac' nerve in man. This has not been re-identified, but Zeller and Burget (1937) suggested that in the dog inhibitory impulses left the cervical vagus and travelled down the wall of the oesophagus, either by way of vagal fibres or by propagation in the intramural plexus. Support for this concept was provided by Grondahl and Haney (1940) and by

\footnotetext{
: Recipient of a traveiling fellowship from the Wellcome Trust. Present address: St. Thomas' Hospital, London.
}

Lehmann (1945), who interrupted these impulses in dogs by oesophageal section.

Although unilateral and supradiaphragmatic bilateral vagotomy are without effect on the dog's inferior gastro-oesophageal sphincter, high bilateral section has been shown to cause a narrowing of the lower part of the oesophagus (Zeller and Burget, 1937 ; Hwang, Essex, and Mann, 1947). Cannon (1907) showed that in the cat the lower part of the oesophagus was still capable of peristalsis even after bilateral vagotomy.

The role of the sympathetic system is disputed. Knight (1934) concluded that sympathetic and vagal nerves were antagonistic in their action on the cat's gastro-oesophageal sphincter and that the resting tone of the latter was maintained by the sympathetics. Carlson, Boyd, and Pearcy (1922), working on a variety of animals, suggested that it was the state of muscular tone at the time of stimulation which determined the response to sympathetic and vagal stimulation. Alnor and Ohnesorge (1958) showed that sympathetic stimulation in the cat and rabbit caused an increase in sphincteric tone, but that the cardia was nevertheless capable of normal function after sympathectomy. Zeller and Burget (1937) agreed that sympathectomy was without effect on the sphincter.

The possibility that the sphincter might be partly autonomous has been considered by several authors. Kronecker and Meltzer (1883) stated that in man the lower par't of the oesophagus exhibits spontaneous activity. Cannon (1907) thought that the lower part of the oesophagus, being smooth muscle, was capable of peristalsis without an extrinsic innervation. Burget and Zeller (1936) showed that sphincteric relaxation in the dog was independent of local extrinsic nervous supply, whereas Alnor and Ohnesorge (1958) thought that the lower portion of the 
oesophagus possessed intrinsic tone which was inhibited by the vagus.

Interpretation of these findings is difficult. As Ingelfinger (1958) pointed out, devices which have been used for the study of sphincteric motility may themselves distort the normal pattern by their very presence. However, the work reported in the literature suggests that the lower portion of the oesophagus is under vagal control, inhibition being mediated by impulses transmitted intramurally.

More recently, Carveth, Schlegel, Code, and Ellis (1962) studied dogs and employed manometric methods. They found that bilateral subhilar vagotomy reduced both the resting tone of the sphincter and the incidence of relaxation in response to deglutition.

The present investigation was undertaken to test the action of the gastro-oesophageal sphincter after sympathetic denervation, after sympathetic denervation combined with vagotomy, or after various oesophageal interruption procedures.

\section{METHODS}

Healthy adult mongrel dogs weighing 10 to $15 \mathrm{~kg}$. were used. The dogs were trained to lie quietly during radiological and manometric studies so that no anaesthesia was necessary.

Fluoroscopic and cineradiographic studies of the oesophagus and gastro-oesophageal sphincteric region were carried out on intact dogs and on all those who had undergone some form of oesophageal interruption. The study was made after the instillation of $30 \mathrm{ml}$. of contrast medium (Micropaque) into the oesophagus through a catheter.

The methods used for the manometric studies were similar to those previously described by Schlegel and Code (1958). Intraluminal pressures were detected by three or four small-calibre water-filled polyethylene tubes connected proximally to strain gauges (Statham, Model P-23De) and recorded photokymographically. The open tip of the distal tube was covered with a small latex balloon $5 \mathrm{~mm}$. in diameter, as described by Code and Schlegel (1958), and lateral orifices were cut in each of the other tubes at intervals of $5 \mathrm{~cm}$. (Fig. 1). During tests the balloon and all the tubes were filled with water and connected to externally placed pressure transducers. To achieve some degree of rigidity, a fine wire within an extra tube was incorporated into the unit. Pressures were recorded under resting conditions at intervals of $0.5 \mathrm{~cm}$. as the tubes were withdrawn from the stomach through the gastrooesophageal sphincter region and into the oesophagus. Responses to deglutition initiated by the injection of a small quantity of water into the pharynx were also recorded at similar intervals through the sphincter. Respiratory movements were recorded by a belt pneumograph fastened around the thorax of each animal and connected to another strain gauge.

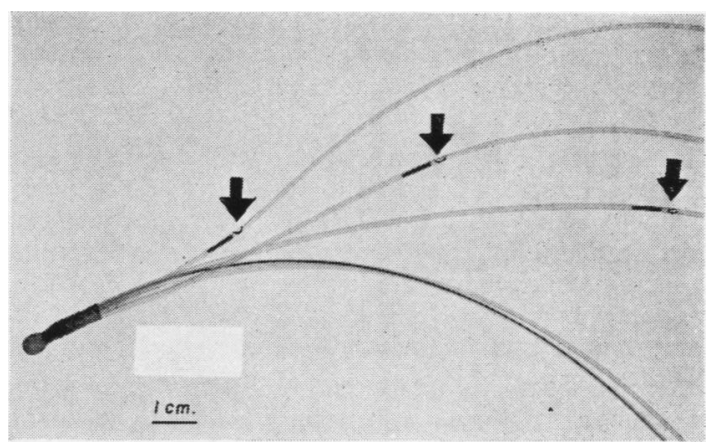

FIG. 1. Pressure-detecting probes employed in manometric studies. The arrows indicate the lateral orifices of the tubes referred to as open-tip tubes.

ANALYSIS OF Records.-Analysis of the manometric records was basically the same as that described by Fyke, Code, and Schlegel (1956). The resting pressures were measured at end-expiratory and endinspiratory phases of respiration and referred to mean gastric fundic pressures, which were arbitrarily designated as zero. The point on inspiration where the pressure deflection changed from positive to negative was noted and referred to as the "respiratory reversal" point. When more than one test was carried out on any dog the mean values were calculated. In a group of dogs which had undergone the same procedure, the mean, standard deviation, and standard error of the mean were calculated. Differences between pre-operative and post-operative values were compared statistically using Student's $t$ test for the significance of the differences (Fisher, 1930).

Duration, maximal amplitude, and time from the instant of swallowing to the onset and the peak of deglutitive responses were measured. In addition, the incidence of sphincteric relaxation and contraction in response to swallowing was determined in the preoperative and post-operative studies.

Operative Procedures.-All operative procedures were carried out with the animal under ether anaesthesia.

Radical Sympathectomy.-In seven dogs an extensive sympathetic denervation of the cardiac end of the stomach, including the gastro-oesophageal sphincteric zone, was effected. Through a left thoraco-abdominal approach the left thoracic sympathetic chain and ganglia were removed from the level of the eighth rib to the diaphragm. The solitary splanchnic nerve arising from the chain at the level of the thirteenth rib also was removed. The right sympathetic chain was exposed by mobilizing the aorta ; it was divided and a strip $2 \mathrm{~cm}$. wide was excised from its lower thoracic part. The right and left coeliac ganglia, which were enmeshed in a plexus of sympathetic nervous tissue around the coeliac artery, were removed and the adventitia was stripped from the vessel and from the proximal few centimetres of its three 
branches. The left gastric artery was divided near its origin to interrupt any nerve fibres which had escaped the above ablations (Fig. 2 A, A', B, C).

Other Sympathetic Denervations.-In one dog, division of the left gastric artery was combined with gastric transection $2 \mathrm{~cm}$. below the gastro-oesophageal sphincter (Fig. 2 C, D). Gastric transection was performed to cut any sympathetic fibres within the stomach wall. It was also carried out as a secondary manoeuvre in one of the dogs on which sympathectomy had been performed previously (Fig. 2 D).

Sympathectomy and Vagotomy.-At a second stage, in four of the sympathectomized dogs a bilateral subhilar thoracic vagotomy was done through a right thoracotomy approach (Fig. 2 E, E'). At this second operation it was confirmed that the right sympathetic chain had been interrupted. The remainder of the chain and splanchnic nerve were removed down to the diaphragm (Fig. $2 \mathrm{~A}^{\prime}$ ).

Oesophageal Interruption.-Three varieties of oesophageal interruption were used. A low oesophageal transection $0.5 \mathrm{~cm}$. above the sphincter was performed in one dog (Fig. $3 \mathrm{~A}$ ). In another animal a $2 \frac{1}{2}$ to $3 \mathrm{~cm}$. segment of jejunum on its vascular pedicle was raised into the thorax and inserted antiperistaltically into the lower part of the oesophagus by two end-to-end anastomoses after excision of an appropriate segment of oesophagus (Fig. $3 \mathrm{~B}$ ). In both of these animals a vagal oesophageal branch was resected to permit of adequate mobilization of the oesophagus.

In the remaining six dogs of this series, an artificial prosthesis was inserted into the lower part of the oesophagus (Fig. $3 \mathrm{C}$ ). Two types of prosthesis were used: one was made of an outer layer of knitted, crimped Teflon and an inner layer of Silastic; the other was made of two layers of crimped Tefion. The prostheses were of varying lengths $(1$ to $5 \mathrm{~cm}$.)

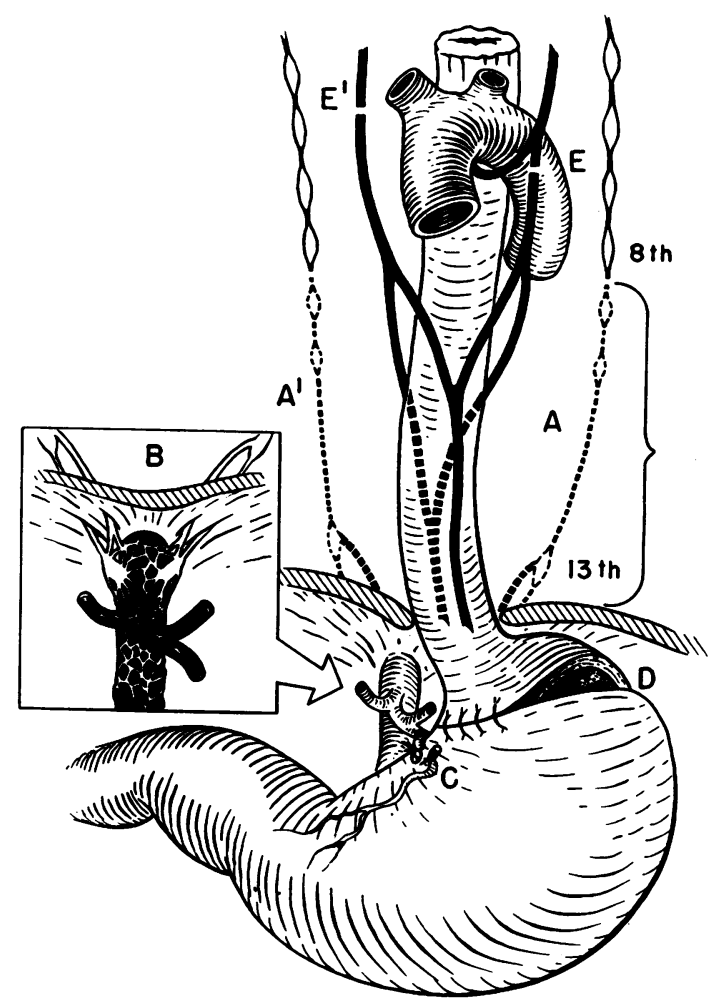

FIG. 2. Diagram of denervation procedures: $A$ and $A^{\prime}$, removal of thoracic sympathetic chain and ganglia within area indicated by bracket; $B$, splanchnicectomy and coeliac gang.ionectomy; $C$, division of left gastric artery; $D$, gastric transection; $E$ and $E^{\prime}$, subhilar bilateral vagotomy.
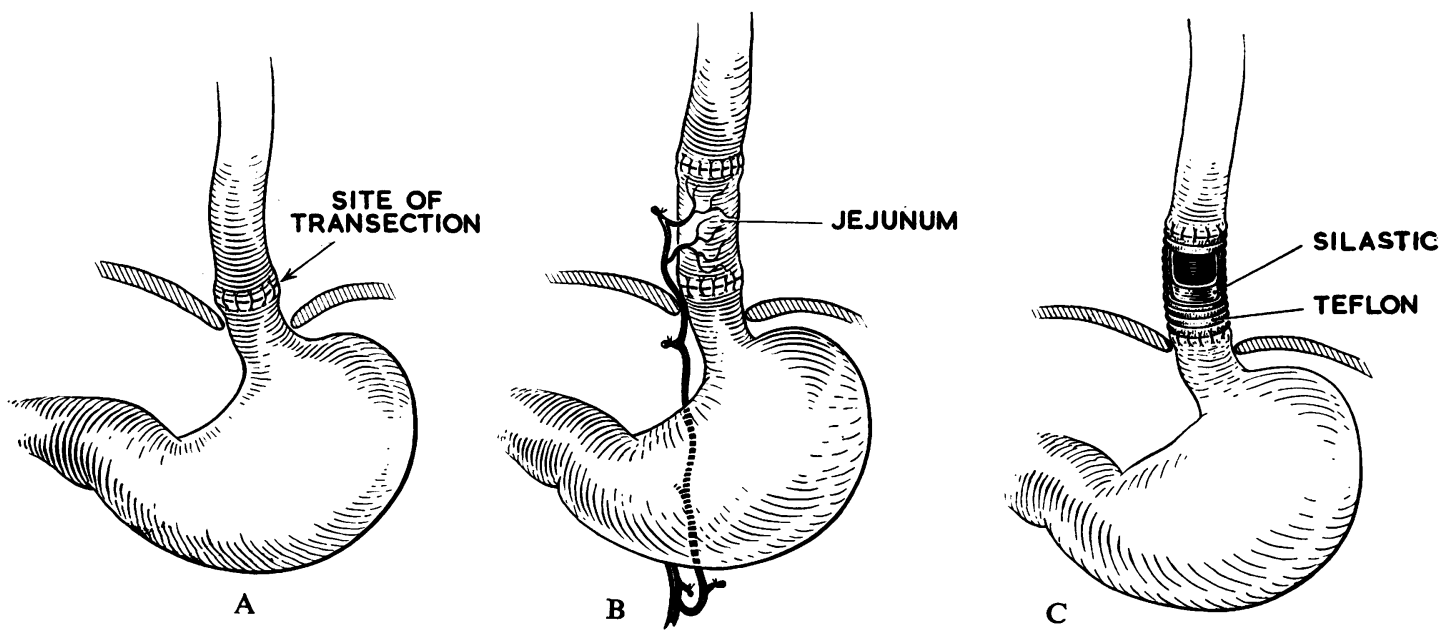

FIG. 3. Diagram of oesophageal interruption procedures: $A$, oesophageal transection; $B$, jejunal prosthesis; and $C$, artificial prosthesis. 
and were inserted at different levels in the lower part of the oesophagus, appropriate segments of the latter being removed to accommodate them. The prostheses were inserted with two end-to-end anastomoses and with varying degrees of vagal resection from minimal oesophageal branch division to total bilateral subhilar vagotomy.

\section{RESULTS}

Control Observations. - During the preoperative period the dogs were healthy, had no dysphagia, and did not lose weight.
No dilatation of the oesophagus was seen in the cineradiograph of any of the intact dogs, and in each the radio-opaque material reached the lower end of the oesophagus rapidly and passed through the sphincter without delay (Fig. 4).

Resting manometric studies were performed on 31 intact dogs, each having one or more tests. As the units were withdrawn from the stomach to the oesophagus, the gastro-oesophageal sphincter was always identified as a zone of raised pressure (Figs. 4 and 5).
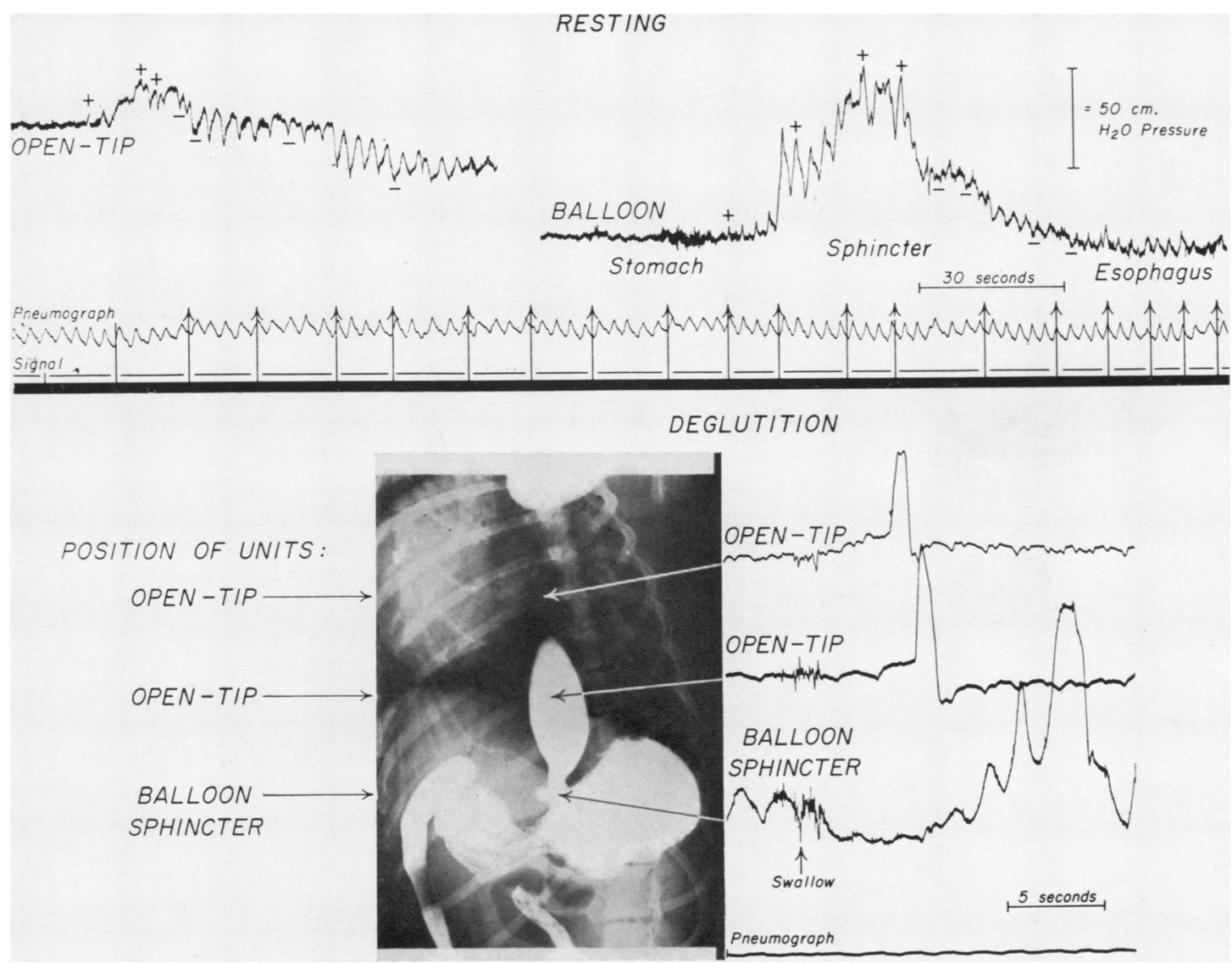

FIG. 4. Resting and deglutition pressures at the gastro-oesophageal sphincter of an intact dog. Upper panel: Resting pressure detected by an open-tip and balloon-tip tube: (+) increase of pressure with inspiration; (-) decrease of pressure with inspiration; each upright arrow signifies $a 0.5 \mathrm{~cm}$. oral withdrawal of the detecting units as they were withdrawn from the stomach into the oesophagus. Note the sharp change in the direction of the pressure swings from (+) to (-) with inspiration and the distinct rise of pressure as the units passed through the sphincter. Lower right panel: Pressure changes after deglutition. Note the peristaltic sequence in the oesophagus and that the pressure in the sphincter declines and remains lowered until the wave of deglutition reaches it; the sphincter then closes with a prolonged contraction. Lower left panel: No evidence of delay in the passage of the contrast medium through the oesophagus or sphincter was noted. 
Deglutition studies were made on 12 dogs. Each swallow was followed by a sphincteric response of relaxation followed by a phase of contraction (Fig. 4). Relaxation commenced 2.6 sec. after deglutition, lasted $7.8 \mathrm{sec}$, and was followed by a phase of contraction lasting $6.9 \mathrm{sec}$. (mean values).

Post-operative Observations.-Studies were made as early as 24 hours and as late as six months after operation; the majority, however, were carried out during the first three postoperative weeks.

Radical Sympathectomy.-Seven dogs were studied after radical sympathectomy. Their clinical post-operative course was without incident; they remained well for a significant period and eventually were studied after further interruption procedures.

The resting pressure profile of the gastro-oesophageal sphincter and its responses to swallowing
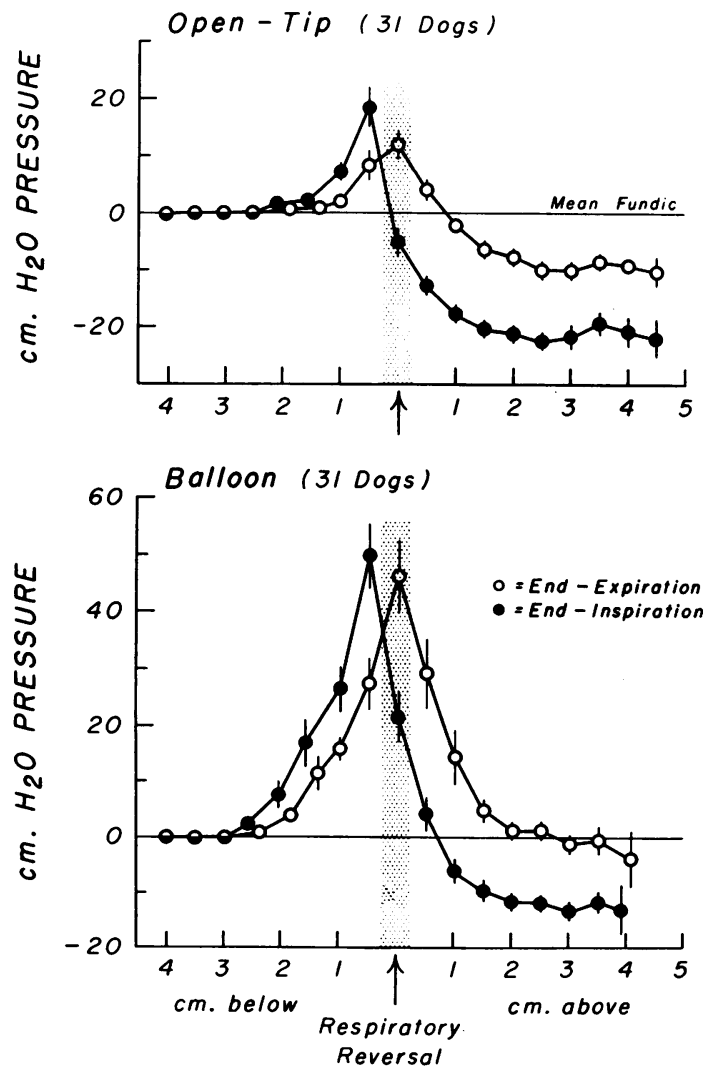

FIG. 5. Mean resting pressure profile of the gastrooesophageal junction of 31 intact dogs. $\dot{i}-\dot{\phi}=$ mean and S.E. of mean. were not affected significantly by sympathectomy (Fig. 6, left).

Other Sympathectomy Procedures. - The clinical course and results of the manometric tests in the two dogs that had an upper gastric transection were essentially the same as those in the larger sympathectomy series. Neither the resting profile nor the responses of the sphincter to deglutition were altered by the operation.

Sympathectomy and VAgotomy. - Bilateral subhilar vagotomy was performed in four of the sympathectomized dogs. After vagotomy all of the animals regurgitated food and lost weight.

Manometric studies revealed a definite band of raised pressure persisting between the stomach and the oesophagus in each of the four dogs (Fig. 6 , right). The amplitude of the resting pressure in the sphincter, however, was less than before the vagotomy. In contrast, the resting pressures in the lower part of the oesophagus were increased after vagotomy.

Deglutition studies after vagotomy showed that the sphincter still relaxed and contracted (Fig. 6, right), but the incidence of these responses, particularly relaxation, was reduced. The range of the pressure changes during those responses which did occur was normal. The onset of contraction in the sphincter above the level of the hiatus was premature, and the duration and amplitude were reduced, the alteration as detected by open tip being highly significant $(P>0.01)$.

While the animals were still healthy, each was given an overdose of anaesthetic and necropsy was performed. The oesophagus of each animal was found to be dilated, and this dilatation probably accounted for the increased resting pressure in the lower part of the oesophagus.

Oesophageal Interruption Procedures. Clinically, all dogs that had undergone some form of oesophageal interruption exhibited marked dysphagia with regurgitation of food. At necropsy organic stricture was not found at the site of operation in the dogs that had undergone transection or in those with jejunal transplant. One of the dogs with an artificial prosthesis survived for several months. At necropsy no sign of the prosthesis could be found. A stricture $1 \mathrm{~cm}$. in diameter had developed at the site of its insertion.

In the cineradiographic studies, the animal that had undergone low transection showed retention of contrast medium in the lower part of the oesophagus without dilatation (Fig. 7). The sphincter did relax from time to time, allowing passage of material into the stomach. In the animal with a jejunal transplant, obstruction 


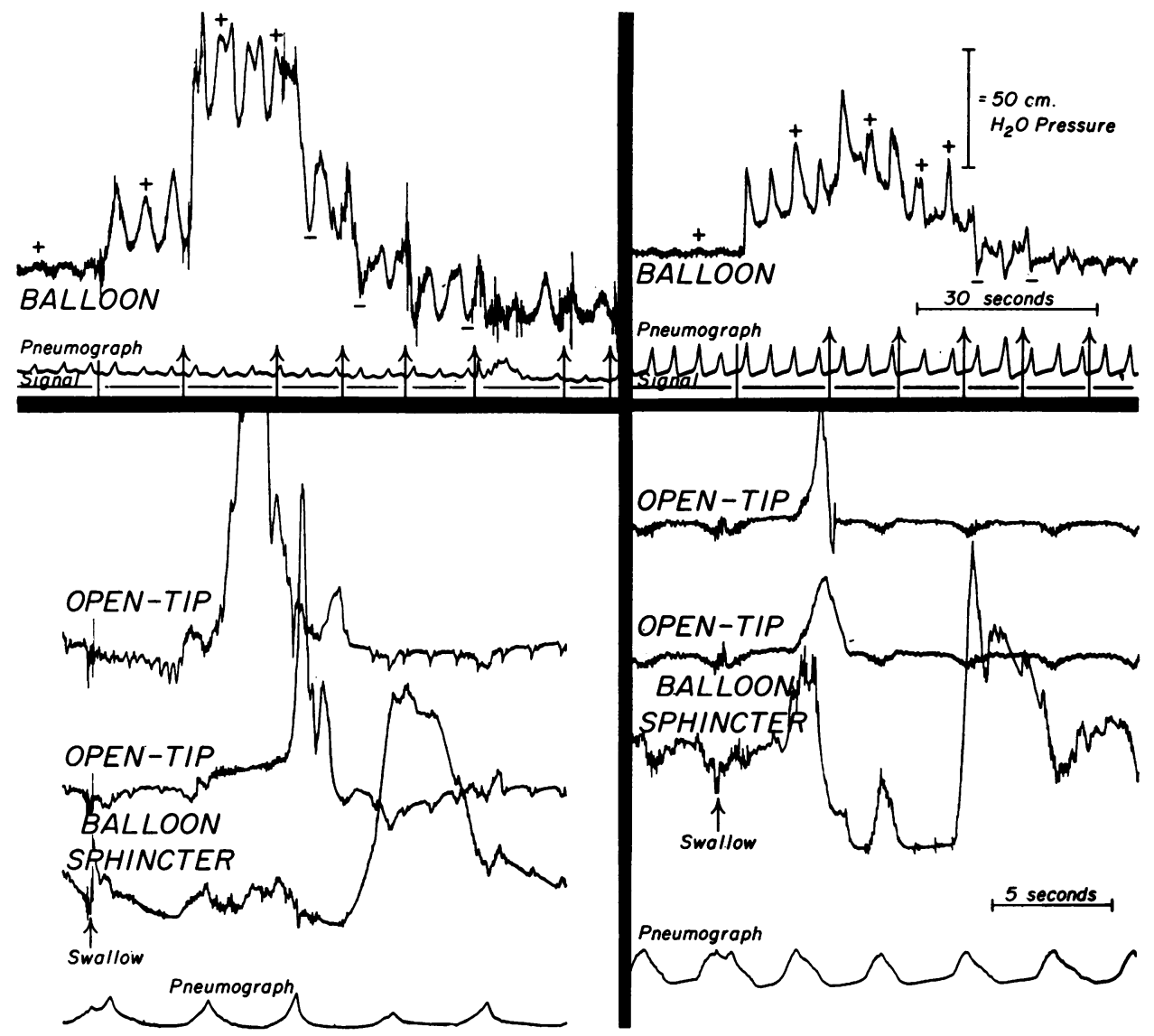

FIG. 6. Resting and deglutition pressures at the gastro-oesophageal sphincter after sympathectomy and sympathectomy plus bilateral subhilar vagotomy. Left panels: Sympathectomy alone was without effect upon the resting pressure and deglutition response of the sphincter. Right panels: After the addition of vagotomy the resting pressure at the sphincter was lowered and that in the oesophagus raised. With deglutition oesophageal complexes were simultaneous. Note, however, that the sphincter relaxed. The pattern is similar to that after vagotomy alone.

developed at the site of the transplant and also at the site of the gastro-oesophageal sphincter. The oesophagus proximal to each was dilated. Continuous tonic activity in the jejunum and considerable delay in the lower part of the oesophagus were noted before the sphincter relaxed to allow emptying (Fig. 8). In each dog with an artificial prosthesis, no delay in the passage of contrast medium was seen at the level of the prosthesis but considerable delay occurred in the lower part of the oesophagus where the degree of dilatation was variable (Fig. 9). The sphincter in these dogs also relaxed from time to time to allow oesophageal emptying.

Manometric findings were the same in this series of dogs as in the animals that had undergone vagotomy. The resting pressure of the gastrooesophageal sphincter was reduced and that in the oesophagus just proximal to the sphincter was increased (Figs. 7, 8, and 9). Sphincteric responses to deglutition also were reduced, particularly relaxation. The responses which did occur were qualitatively normal with the exception of suprahiatal contraction, which was diminished.

\section{Discussion}

Although the sympathetic innervation of the canine gastro-oesophageal sphincter is not well defined, it is said to arise from below the diaphragm, originating in the coeliac ganglion and being distributed via the coeliac plexus along the trunk and terminal branches of the left gastric 

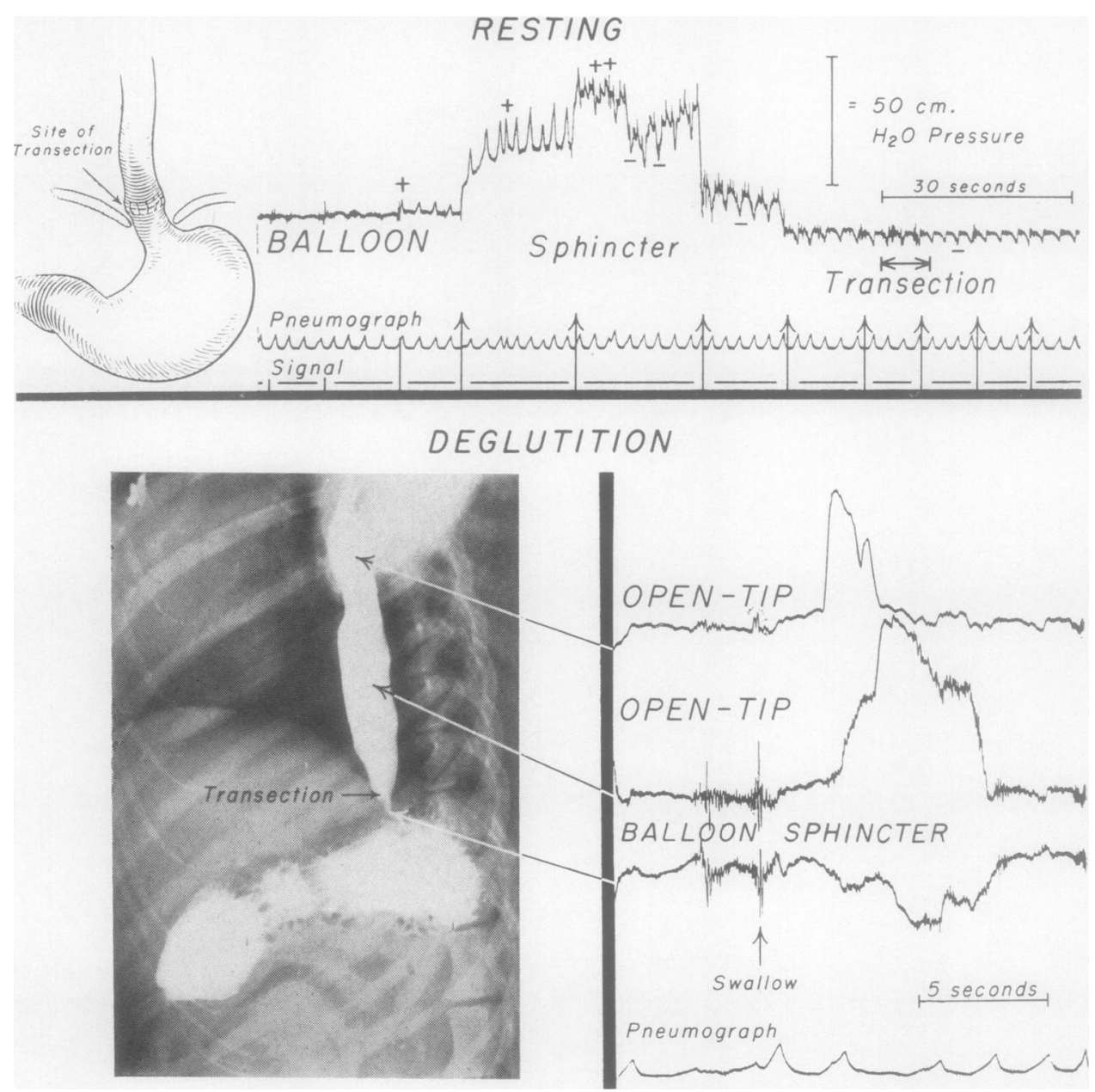

FIG. 7. Pressures at the gastro-oesophageal junction after transection and resuture of the lower part of the oesophagus. Upper right panel : Resting pressure profile contains a zone of raised pressure normal in amplitude and contour. Lower right panel: With deglutition a peristaltic wave traversed the oesophagus and normal relaxation occurred within the sphincteric region. Lower left panel: A radiograph revealed evidence of delay in the passage of the radio-opaque material through the oesophagus.

artery (Knight, 1934 ; Alnor and Ohnesorge, 1958 ; McCrea, 1926; and Mizers, 1955). Coeliac ganglionectomy combined with left gastric division should interrupt these fibres; however, in this study a more radical procedure was performed to ensure the severance of all sympathetic fibres to the sphincteric zone. In addition to coeliac ganglionectomy, interruption and removal of both lower thoracic sympathetic chains and splanchnic nerves were performed. To divide any possible fibres within the stomach wall which might have escaped the other ablations, the upper part of the stomach was transected at a later stage in one dog, and this latter procedure was combined with left gastric division in another animal. These sympathetic denervations did not affect the function of the canine gastro-oesophageal sphincter, and the conclusion is justified that this sphincter operates independently of the sympathetic nervous system.

The effect of vagotomy at different levels on the gastro-oesophageal sphincter has recently been studied by Carveth and associates (1962) by means of a similar manometric technique. They showed that whereas bilateral subhilar vagotomy lowered the resting sphincteric pressure barrier between the stomach and the oesophagus and altered its 

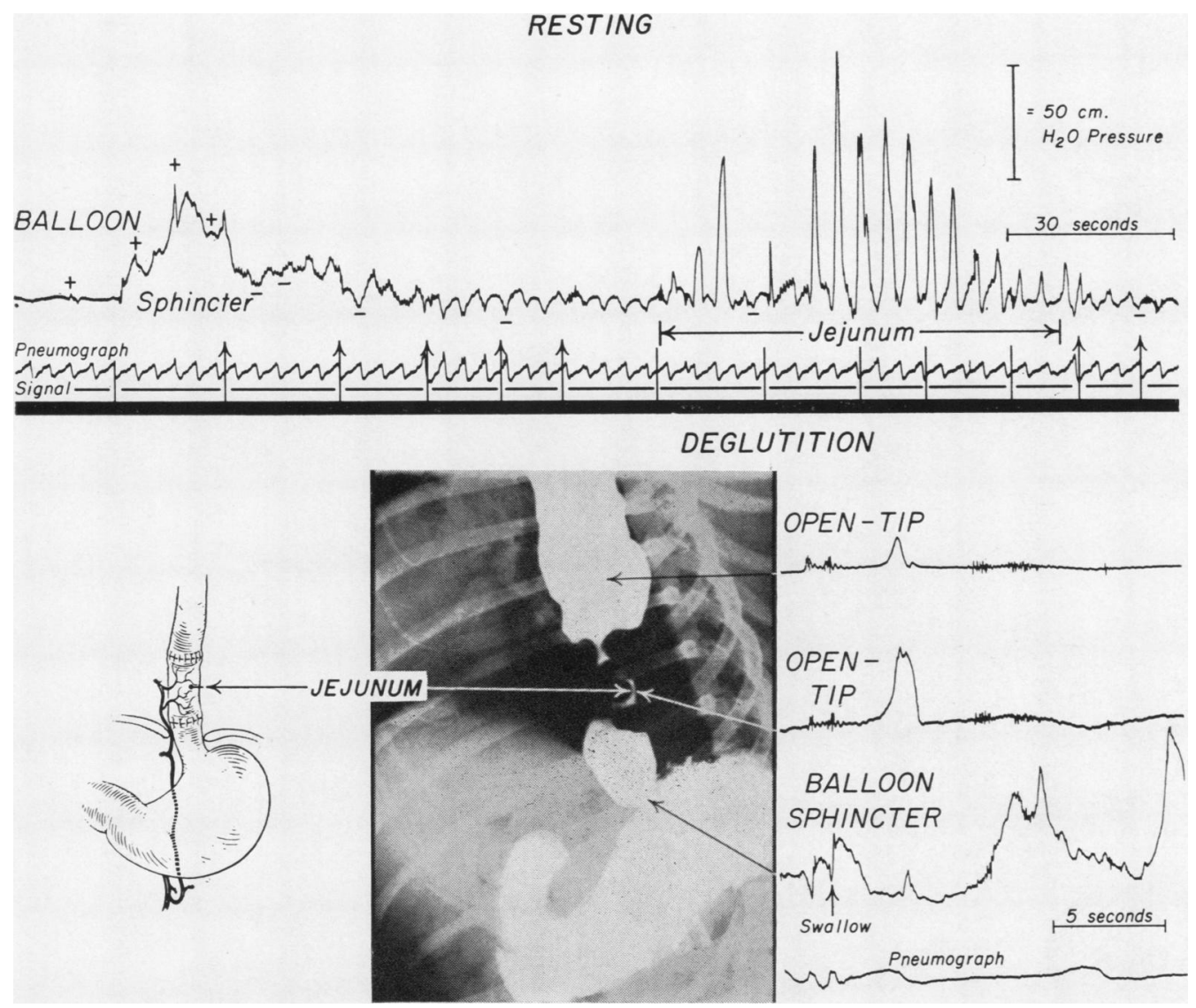

FIG. 8. Intraluminal pressures after interposing a segment of jejunum into the distal oesophagus. Upper panel: Resting pressures. Note the raised pressure at the sphincter and the rhythmical contractions occurring in the jejunum at a rate of 14 to 15 waves/min. Lower right panel: With deglutition the pressures in the oesophagus and jejunum increased simultaneously while the pressure of the sphincter aeclined. Lower mid panel: Contrast radiography revealed a dilated oesophagus above and below the jejunal segment with considerable retention of Micropaque. Note the narrowed segment of jejunum.

responses to deglutition, cervical vagotomy completely abolished the zone of raised pressure and eliminated all response to deglutition. An explanation for these findings could be that fibres in the cervical vagi leave these nerves above the hilum of the lung and travel to the gastro-oesophageal sphincter by a different route, possibly via the sympathetic system.

In the present study, bilateral subhilar vagotomy was combined with radical sympathectomy. In theory this procedure should eliminate all extrinsic autonomic nervous supply to the gastrooesophageal sphincter and should reveal whether the action of the vagus is in any way dependent on the integrity of the sympathetic system. Bilateral subhilar vagotomy in animals that had already undergone sympathectomy produced results similar to or identical with those previously reported for bilateral subhilar vagotomy alone. The dogs suffered from dysphagia and regurgitation. Manometrically, the resting sphincteric pressure was reduced but not eliminated. The incidence of sphincteric responses to deglutition was reduced, but those that occurred were normal except for diminished contraction above the hiatus. Thus sphincteric function was not abolished by the combination of sympathectomy with subhilar vagotomy. This result forces the conclusion that other nerve fibres must reach the sphincteric area by routes not affected by these 

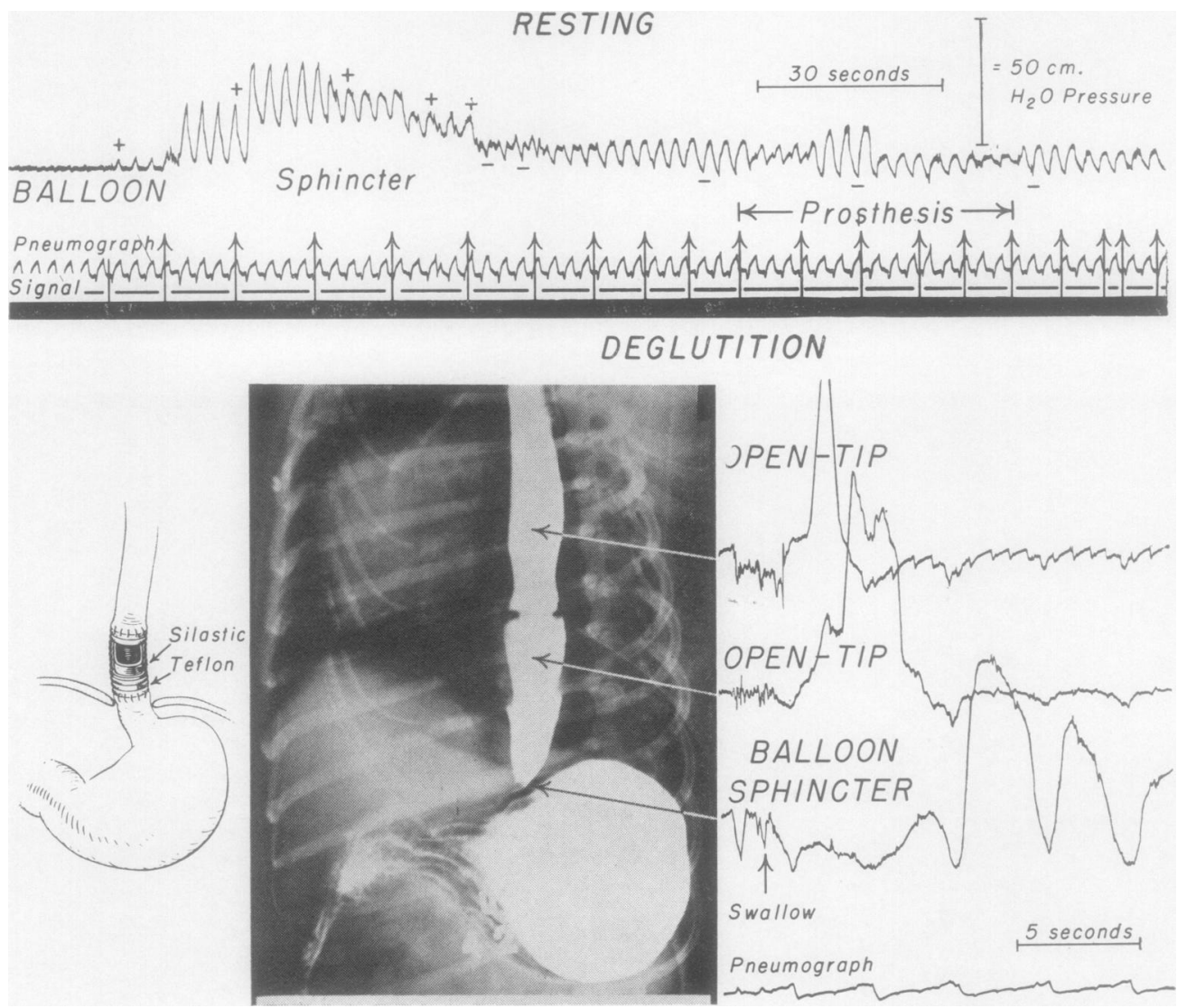

FIG. 9. A segment of oesophagus is replaced by an artificial prosthesis. Upper panel: Resting pressure profile contains a zone of raised pressure which is within normal limits. Lower right panel: With deglutition, a peristaltic contraction progressed through the oesophagus and the sphincter relaxed and contracted normally. Lower mid panel: Some retention of contrast medium and slight dilatation of the oesophagus are evident radiographically. The prosthesis is clearly defined.

ablative procedures or else the gastro-oesophageal sphincter enjoys a considerable degree of autonomy.

A possible additional route of access of extrinsic nerve fibres to the sphincter is down and in the wall of the oesophagus, either as actual nerves or as propagated impulses within the intramural plexuses. Such a route has been proposed by Zeller and Burget (1937). Oesophageal transection would be an effective method of interrupting such nerve fibres or impulses. This has been performed alone or in combination with vagotomy with divergent results. Grondahl and Haney (1940) and Carveth and associates (1962) found muscle girding or transection alone to be without effect. Lehmann (1945) noted that it abolished sphincteric relaxation. When thoracic vagotomy was added to the procedure, Grondahl and Haney (1940) found that sphincteric relaxation was prevented, but Carveth and associates (1962) noted that relaxation persisted. In the present study, oesophageal transection was performed as low as possible in order to achieve maximal isolation of the sphincter and to remove any immediately proximal oesophageal segment in which a peristaltic wave might be initiated. The regurgitation, delay in the radiograph, and manometric findings which this dog showed were undoubtedly attributable to the interruption of the vagal branch which accompanied the transection. The sphincter itself behaved nearly normally.

In view of the possibility that a peristaltic impulse might " jump" across a short area at the site of a simple transection, or that the severed nerve and 
muscle fibres might quickly reunite, it was decided to excise a segment of oesophagus and interpose a prosthesis in order to interrupt oesophageal continuity more effectively. A living autograft, a segment of jejunum, was chosen initially. It was inserted retrogradely so that the jejunum would be working in the opposite direction to oesophageal peristalsis. The clinical course, cineradiographic studies, and necropsy findings showed that this occurred, there being a functional obstruction at the level of the prosthesis for which no organic cause could be found. Apart from the obstruction so induced, the findings in this animal were similar to those found in the low transection, a band of raised pressure capable of relaxation still persisting in the sphincteric region.

As a final stage in this series of experiments a foreign rigid prosthesis was interposed in the oesophagus to effect the interruption, thus eliminating any remote possibility of " reverse peristalsis " that might have been established in the living autograft. Differing degrees of coincident vagotomy also were affected ; in some cases only minimal division of oesophageal branch resection was performed, in others total bilateral subhilar vagotomy was carried out. Therefore, the effect of these vagal interruptions was added to the presence of the prosthesis. The findings in these dogs were the same as in those with vagal interruption alone, namely, oesophageal delay, reduced resting sphincteric pressure, and a sphincter that relaxed and contracted with relatively normal character but reduced incidence.

From the observations made on these animals, the concept that complete control of the gastrooesophageal sphincter is mediated by nerve fibres or impulses coursing for some distance down the wall of the oesophagus is no longer tenable. These results combined with those of the denervation studies suggest that the gastro-oesophageal sphincter can function in the absence of extrinsic nerve supply. The data indicate that while there is no doubt that the vagus nerve exerts some influence over the canine gastro-oesophageal sphincter, the sphincter nevertheless enjoys a degree of autonomy that is independent of any local extrinsic nerve supply. Such a hypothesis is not new (Kronecker and Meltzer, 1883; Cannon, 1907 ; Burget and Zeller, 1936; and Alnor and Ohnesorge, 1958); our data simply strengthen its likely truth. Why and how the denervated sphincter maintains an inherent resting tone is obscure, but it is possible that the occurrence of relaxation, when a bolus presents from above, may be mediated by the local autonomic nerve plexuses within the structure of the sphincteric region itself, perhaps stimulated by the distending force of the bolus (Creamer and Schlegel, 1957). Alternatively, the action may be a property of the sphincteric musculature.

\section{Summary}

The effect of certain nerve and oesophageal interruption procedures on the function of the canine gastro-oesophageal sphincter has been studied clinically, radiographically, and, more particularly, by manometric determination of oesophageal and sphincteric motility, both in the resting phase and on deglutition.

Radical sympathetic extirpation was without effect.

Radical sympathetic extirpation combined with bilateral subhilar vagotomy has an effect similar to that of subhilar vagotomy alone, that is, diminution of resting sphincteric tone with reduced incidence of sphincteric response to deglutition, particularly relaxation.

Various oesophageal interruptions and interpositions have a relatively minor effect on the sphincter, the changes depending entirely on the degree of concomitant vagotomy carried out.

A band of raised pressure capable of relaxation and contraction persisted in the sphincteric area after all of these procedures. The conclusion has been drawn that the canine gastro-oesophageal sphincter can relax and contract in co-ordination with swallowing independently of local extrinsic nerves and that it thus enjoys a considerable degree of autonomy.

This investigation was supported in part by research grants A-2015 and A-5457 from the National Institutes of Health, U.S. Public Health Service.

\section{REFERENCES}

Alnor, P.C., and Ohnesorge, F. K.(1958). Z.ges. exp. Med., $129,511$. Burget, G. E., and Zeller, W. E. (1936). Proc. Soc. exp. Biol. (N.Y.), Cannon, W. B. (1907). Amer. J. Physiol., 19, 436.

Carlson, A. J., Boyd, T. E., and Pearcy, J. F. (1922). Amer. J. Physiol., 61, 14.

Carveth. S. W., Schlegel, J. F., Code, C. F., and Ellis, F. H., Jr. (1962). Surg. Gynec. Obstet. 114, 31

Code, C. F., and Schlegel, J. F. (1958). Proc. Mayo Clin., 33. 406

Creamer. B., and Schlegel, J. (1957). J. appl. Physiol., 10, 498.

Fisher, R. A. (1930). Statistical Methods for Research Workers, 3rd ed. Oliver and Boyd, Edinburgh.

Fyke, F. E., Jr., Code, C. F., and Schlegel, J. F. (1956). Gastroenterologia, Basel, 86, 135.

Grondahl, J. W., and Haney, H. F. (1940). Proc. Soc. exp. Biol. $(N . Y), 44,126$.

Hwang, Kao, Essex, H. E., and Mann, F. C. (1947). Amer. J. Physiol., 149, 429.

Ingelfinger, F. J. (1958). Physiol. Rev., 38, 533.

Knight. G. C. (1934). Brit. J. Surg., 22. 155.

Kronecker, H.. and Meltzer, S. J. (1883). Arch. Anat. Physiol., Suppl.-Bd. (Physiol. Abt.), p. 328.

Lehmann, G. (1945). Amer. J. Physiol., 143, 163.

McCrea, E. D'Arcy (1926). Brit. J. Surg.. 13, 621

Mizers, N. J. (1955). Amer. J. Anat., 96, 285.

Openchnwski, T. von (1889). Arch. Anat. Physiol. (Physiol. Abt.), p. 549.

Schlegel, J. F., and Code, C. F. (1958). Amer. J. Physiol., 193, 9. Zeller, W., and Burget, G. E. (1937). Amer. J. dig. Dis., 4, 113. 